\title{
A Smartphone Application as an Exploratory Endpoint in a Phase 3 Parkinson's Disease Clinical Trial: A Pilot Study
}

\author{
Alex Page $^{a, b} \quad$ Norman Yung $^{a}$ Peggy Auinger ${ }^{a, b} \quad$ Charles Venuto ${ }^{a, b}$ \\ Alistair Glidden ${ }^{a}$ Eric Macklin ${ }^{c} \quad$ Larsson Omberg $^{d} \quad$ Michael A. Schwarzschild $^{\mathrm{e}}$ \\ E. Ray Dorsey ${ }^{\mathrm{a}, \mathrm{b}}$ \\ ${ }^{a}$ Center for Health + Technology, University of Rochester Medical Center, Rochester, NY, USA; ${ }^{b}$ Department of \\ Neurology, University of Rochester Medical Center, Rochester, NY, USA; 'Department of Medicine, Massachusetts \\ General Hospital, Boston, MA, USA; ${ }^{d}$ Sage Bionetworks, Seattle, WA, USA; 'Department of Neurology, \\ Massachusetts General Hospital, Boston, MA, USA
}

\section{Keywords}

Smartphone $\cdot$ Parkinson disease $\cdot$ Clinical trial $\cdot$ Inosine $\cdot$

Telemedicine · Gait · Movement · Cognition

\section{Abstract}

Background: Smartphones can generate objective measures of Parkinson's disease (PD) and supplement traditional in-person rating scales. However, smartphone use in clinical trials has been limited. Objective: This study aimed to determine the feasibility of introducing a smartphone research application into a PD clinical trial and to evaluate the resulting measures. Methods: A smartphone application was introduced part-way into a phase 3 randomized clinical trial of inosine. The application included finger tapping, gait, and cognition tests, and participants were asked to complete an assessment battery at home and in clinic alongside the Movement Disorder Society-Unified Parkinson's Disease Rating Scale (MDS-UPDRS). Results: Of 236 eligible participants in the parent study, 88 (37\%) consented to participate, and 59 (27 randomized to inosine and 32 to placebo) completed a baseline smartphone assessment. These 59 participants collectively completed 1,292 batteries of assessments.
The proportion of participants who completed at least one smartphone assessment was $61 \%$ at $3,54 \%$ at 6 , and $35 \%$ at 12 months. Finger tapping speed correlated weakly with the part III motor portion ( $r=-0.16$, left hand; $r=-0.04$, right hand) and total ( $r=-0.14)$ MDS-UPDRS. Gait speed correlated better with the same measures $(r=-0.25$, part III motor; $r=-0.34$, total). Over 6 months, finger tapping speed, gait speed, and memory scores did not differ between those randomized to active drug or placebo. Conclusions: Introducing a smartphone application midway into a phase 3 clinical trial was challenging. Measures of bradykinesia and gait speed correlated modestly with traditional outcomes and were consistent with the study's overall findings, which found no benefit of the active drug.

(c) 2022 The Author(s).

Published by S. Karger AG, Basel

\section{Introduction}

Current measures of Parkinson's disease motor and nonmotor symptoms rely on subjective scales that are sparsely collected and generally administered in the clinic [1]. By contrast, smartphones and other digital sensors karger@karger.com www.karger.com/dib

Karger $\stackrel{\text { ' }}{=}$

BOPEN ACCESS
(C) 2022 The Author(s)

Published by S. Karger AG, Basel

This is an Open Access article licensed under the Creative Common Attribution-NonCommercial-4.0 International License (CC BY-NC) (http://www.karger.com/Services/OpenAccessLicense), applicable to the online version of the article only. Usage and distribution for commercial purposes requires written permission.
Correspondence to:

E. Ray Dorsey, ray.dorsey@ chet.rochester.edu 


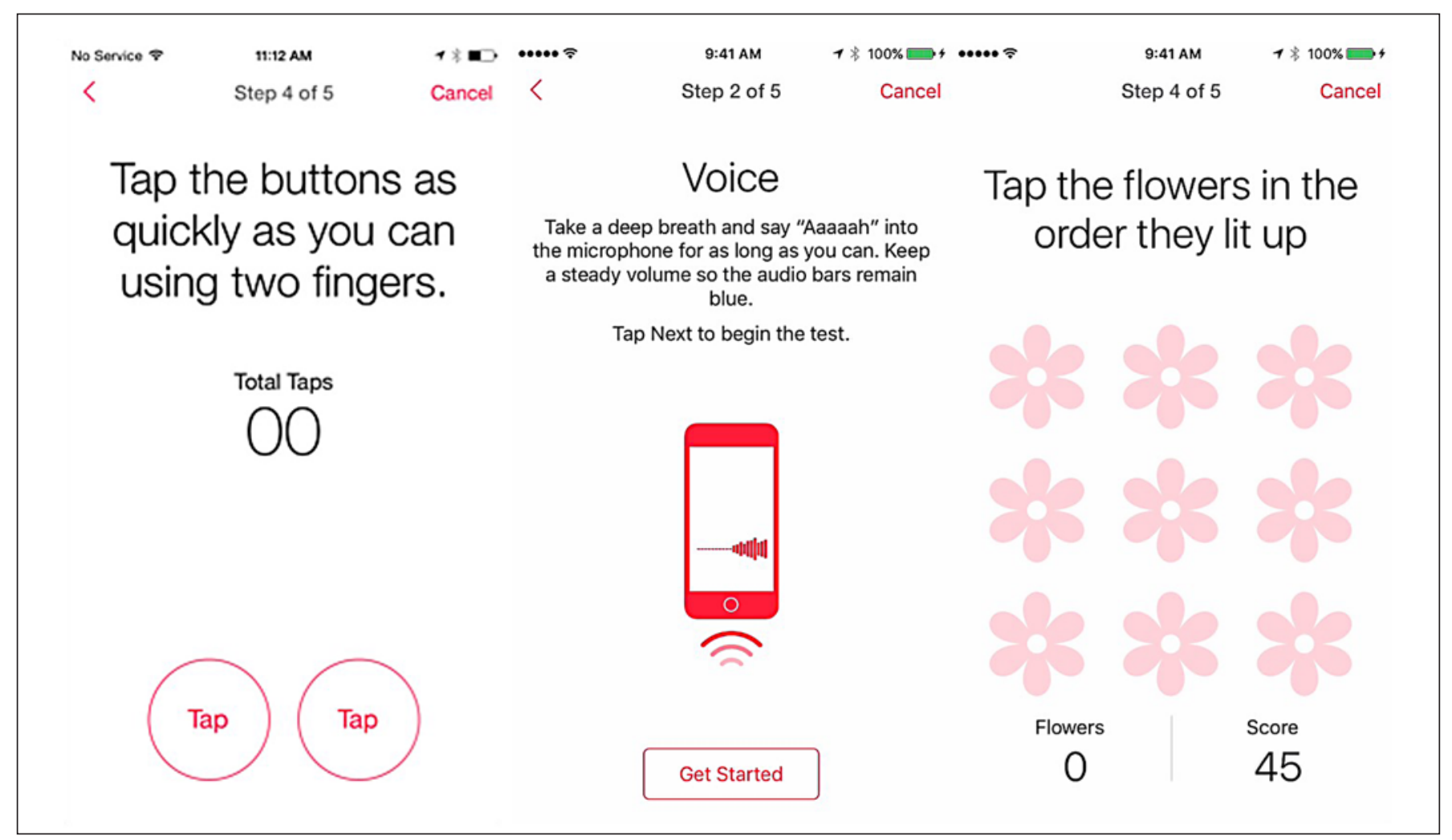

Fig. 1. Examples from the mPower application, including (from left to right) an evaluation of finger tapping, a voice assessment, and a memory test.

can obtain sensitive, frequent, objective measurements of the disease and detect real-world response to proven therapies like levodopa and thus be powerful supplements to current measures $[2,3]$. Recent guidance by regulatory authorities including the US Food and Drug Administration encourages the use of such assessments $[4,5]$. However, while these digital tools have been increasingly studied in observational studies [6-10], the incorporation of smartphones into clinical trials in Parkinson's has been rare $[3,11]$.

The pharmaceutical company Roche has previously developed a smartphone application that incorporates motor tasks, such as finger tapping, that can differentiate individuals with Parkinson's disease from those without [3] and has included it as an exploratory endpoint in a phase 2 clinical trial [12]. We therefore evaluated the feasibility of incorporating an established smartphone application (mPower, by Sage Bionetworks [13]) into a phase 3 Parkinson disease clinical trial [14]. We also sought to determine the correlation of digital measures of bradykinesia, gait, and cognition with traditional outcome measures and their ability to detect any differences between the active drug (inosine) and placebo. The overall aim of this pilot study was to inform the use of digital tools in future clinical trials.

\section{Methods}

\section{Parent Clinical Trial}

This pilot smartphone sub-study was part of a phase 3 randomized, double-blind, placebo-controlled trial of oral inosine [14]. The 2-year, 61-site parent study enrolled 298 participants with early Parkinson's disease not requiring dopaminergic therapy other than an MAO-B inhibitor. Enrollment took place between August 2016 and December 2017. The primary outcome was rate of change in the sum of parts I, II, and III of the MDS-UPDRS until the initiation of dopaminergic therapy. The study was terminated early due to an interim analysis showing that the drug was unlikely to demonstrate efficacy [15].

\section{Smartphone Sub-Study}

An optional sub-study was incorporated into the parent phase 3 clinical trial to evaluate the feasibility and reliability of a smartphone research application to capture and measure disease severity data outside the clinic. The parent and sub-study protocols were approved by the relevant institutional review boards. The initial protocol allowed enrollment into the sub-study prior to random- 


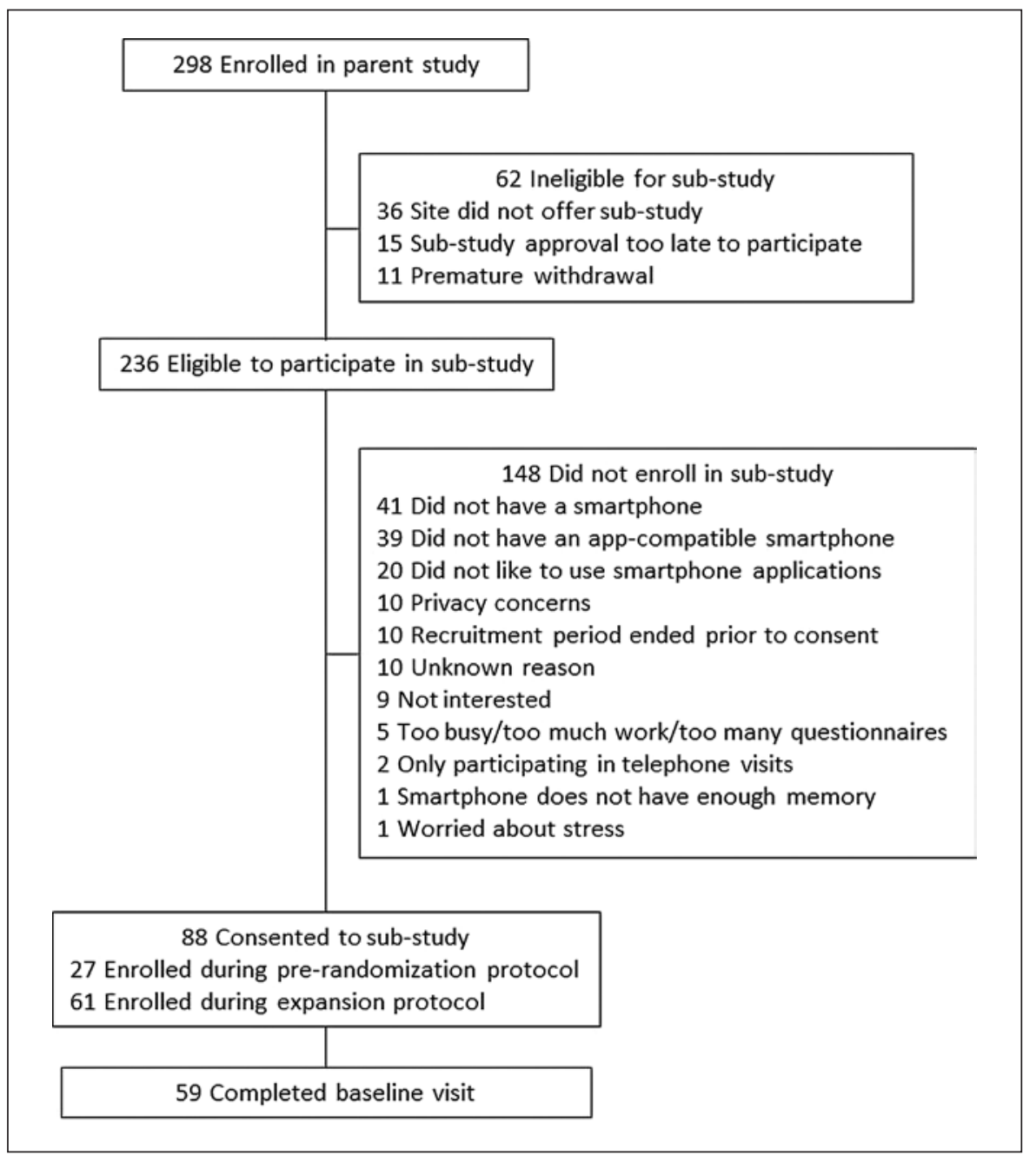

Fig. 2. Participant flow.

ization in the parent study and was later expanded to allow enrollment through the month 21 visit of the parent study. Eligible participants from the parent study included individuals at 36 participating sites who (1) had a smartphone ("bring your own device") with either an iOS or Android operating system that could support the mPower research application and (2) were willing to complete a battery of assessments on the phone at least monthly. The objectives of the pilot study were (1) to evaluate the feasibility of incorporating a smartphone research application into a phase 3 clinical trial; (2) to assess the correlation between measures derived from the smartphone with traditional clinical outcomes assessed in the clinic; and (3) to explore changes in motor and nonmotor function as measured by the smartphone in response to active drug versus placebo relative to traditional, in-clinic measures.

\section{Smartphone Application}

In this study, we customized a smartphone research application (mPower) that has been previously used in an observational study of over 15,000 participants $[13,16]$. The smartphone application had a set of 7 active tasks (finger tapping, standing still for $30 \mathrm{~s}$, walking for $30 \mathrm{~s}$, saying "aaah" for $10 \mathrm{~s}$, assessments of rest and postural tremor, and a brief spatial memory test) designed to assess domains affected by Parkinson's disease. For the finger tapping test, the number of taps completed during each task was calculated, as well as other metrics such as the tapping consistency (variance in tapping intervals) and accuracy (frequency that buttons were missed). Gait parameters were calculated using the PDKit package over 5-s windows of the gait data and averages across windows and repeat measures from individuals $[17,18]$. Finally, the spatial memory test score was calculated by allotting points to each correct sequential response [13]. Participants were asked to complete this approximately 15 -min battery of tests (Fig. 1) at least monthly. Participants received training from the site coordinator on use of the application and could contact the study team via a study-specific email or phone number with questions on use of the application.

\section{Analysis}

Results pertaining to participation (initial enrollment in the smartphone sub-study and completion of an initial smartphone assessment) and retention (completion of an assessment 3, 6, and 12 months after initial use) were analyzed descriptively. Demographic and clinical characteristics reported during the baseline visit of the parent study were compared for those initially enrolled versus not enrolled using $t$ tests for continuous measures and $\chi^{2}$ 
Table 1. Characteristics of the eligible study population by enrollment status

\begin{tabular}{|c|c|c|c|}
\hline & Enrolled $(n=88)$ & Not enrolled $(n=148)$ & $p$ value \\
\hline \multicolumn{4}{|l|}{ Demographic characteristics } \\
\hline Age, yr & $61.7(9.1)$ & $63.1(9.6)$ & 0.26 \\
\hline Female, $n(\%)$ & $49(55.7)$ & $68(45.9)$ & 0.15 \\
\hline Hispanic or Latino ethnicity, $n(\%)(n=234)$ & $2(2.3)$ & $2(1.4)$ & 0.63 \\
\hline \multicolumn{4}{|l|}{ Race, $n(\%)$} \\
\hline White & $83(94.3)$ & $146(98.6)$ & \\
\hline Asian & $1(1.1)$ & $0(0.0)$ & \\
\hline Black or African American & $0(0.0)$ & $2(1.4)$ & $0.11^{\mathrm{a}}$ \\
\hline Multiple race & $2(2.3)$ & $0(0.0)$ & \\
\hline Not specified & $2(2.3)$ & $0(0.0)$ & \\
\hline Education $>12$ years, $n(\%)(n=230)$ & $79(92.9)$ & $133(91.7)$ & 0.74 \\
\hline \multicolumn{4}{|l|}{ Clinical characteristics } \\
\hline Parkinson's disease duration, mo & $9.9(7.8)$ & $10.2(7.8)$ & 0.79 \\
\hline \multicolumn{4}{|l|}{ Modified Hoehn and Yahr, n (\%) } \\
\hline Stage 1 & $24(27.3)$ & $38(25.7)$ & \\
\hline Stage 1.5 & $6(6.8)$ & $10(6.8)$ & \\
\hline Stage 2 & $54(61.4)$ & $98(66.2)$ & $0.79^{\circ}$ \\
\hline Stage 2.5 & $4(4.5)$ & $2(1.3)$ & \\
\hline Modified Schwab and England - ADL $(n=235)$ & $94.2(5.3)$ & $94.2(4.6)$ & 0.98 \\
\hline \multicolumn{4}{|l|}{ MDS-UPDRS } \\
\hline Part I & $5.4(4.0)$ & $5.4(3.6)$ & 0.97 \\
\hline Part II & $5.5(3.9)$ & $5.7(4.3)$ & 0.80 \\
\hline Part III & $21.7(9.3)$ & $22.0(8.7)$ & 0.85 \\
\hline Total I-III & $32.6(12.1)$ & $33.0(12.3)$ & 0.83 \\
\hline Montreal cognitive assessment $(n=235)$ & $27.7(1.7)$ & $27.4(2.0)$ & 0.30 \\
\hline Parkinson's disease questionnaire-39 & $7.9(7.2)$ & $7.5(6.7)$ & 0.70 \\
\hline Neuro-QoL - depression & $42.1(5.5)$ & $41.2(5.4)$ & 0.24 \\
\hline
\end{tabular}

Demographic and clinical characteristics are reported from baseline of the parent study. Results are mean (standard deviation) for continuous measures and $n$ (\%) for categorical measures. Total sample size is reported if there are any missing values. ADL, Activities of Daily Living; MDS-UPDRS, Movement Disorder Society-Unified Parkinson's Disease Rating Scale, Neuro-QOL, Quality of Life in Neurological Disorders. ${ }^{a} p$ value based on Fisher's exact test comparing white versus nonwhite. ${ }^{b} p$ value based on the $x^{2}$ test comparing stages $1-1.5$ versus stages $2-2.5$.

tests or Fisher's exact tests for categorical measures. Given the small sample size and limited retention, we focused our analysis of smartphone assessments on finger tapping, gait, and cognition, especially those conducted in the first 6 months after the first smartphone assessment. Correlations were analyzed using the Spearman correlation coefficient $\left(r_{\mathrm{s}}\right)$ between smartphone assessments and either the nonmotor (part I), motor (part III), or total (parts I-III) MDS-UPDRS score from the participant's most current in-clinic assessment.

\section{Results}

\section{Participants, Use, and Retention}

Of the 298 participants randomized in the parent study, 236 were eligible to participate in the smartphone sub-study, of whom 88 (37\%) consented to participate, starting between February 2017 and October 2018. Of these, 27 (31\%) were enrolled during the pre-randomization protocol, and $59(60 \%)$ completed at least the baseline assessment (Fig. 2). The baseline characteristics of the study participants and nonparticipants are summarized in Table 1. The smartphone application was used in 1,292 sessions. Compliance in the smartphone sub-study declined over time with $61 \%$ at $3,54 \%$ at 6 , and $35 \%$ at 12 months completing at least one smartphone assessment. Twelve participants (20\%) used the app for over 1 year. Older age and a higher MDS-UPDRS total score at baseline were predictive of higher compliance, in agreement with prior work showing higher participation in sicker and older patients [19].

\section{Correlation with Traditional Measures}

Finger tapping speed correlated weakly with the motor (part III: $r_{\mathrm{s}}=-0.16$, left hand; $r_{\mathrm{s}}=-0.04$, right hand) and 


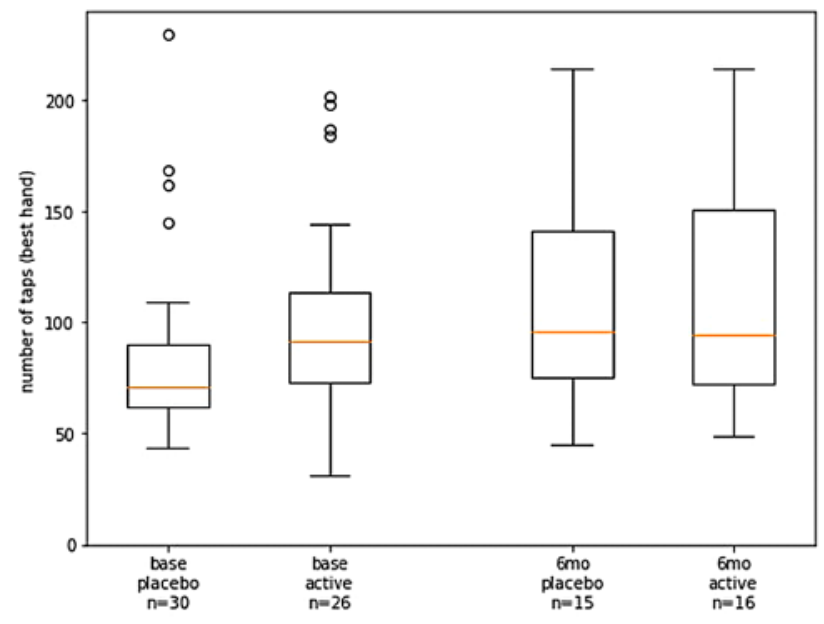

(a) Tapping speed in best hand

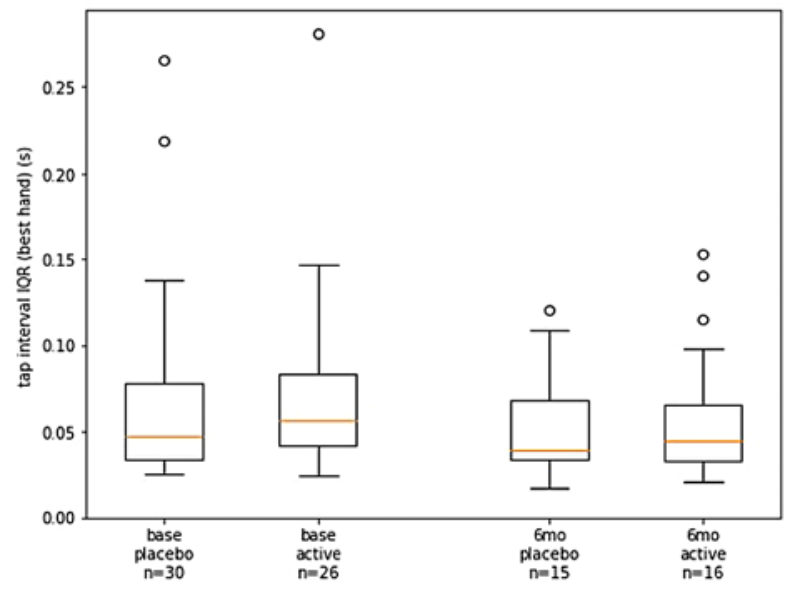

(a) Tapping variability in best hand

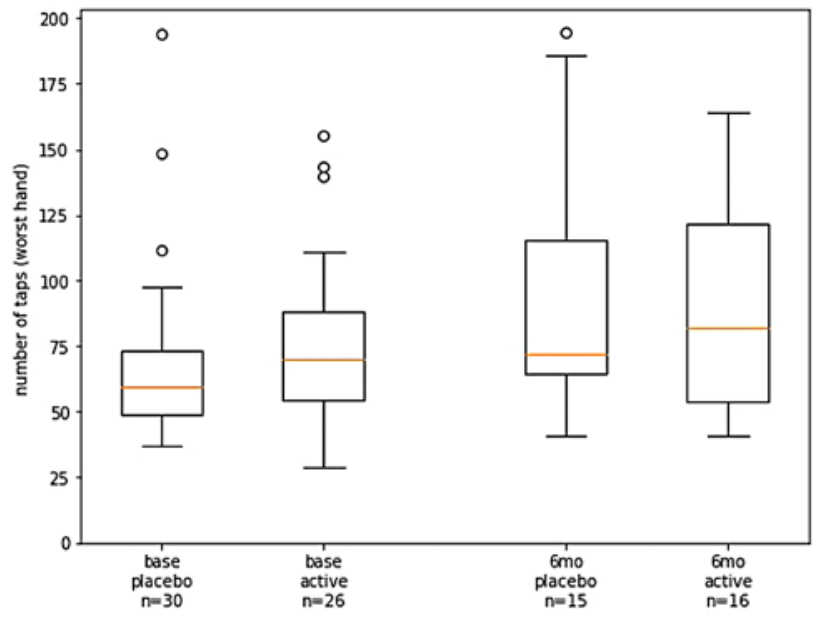

(b) Tapping speed in worst hand

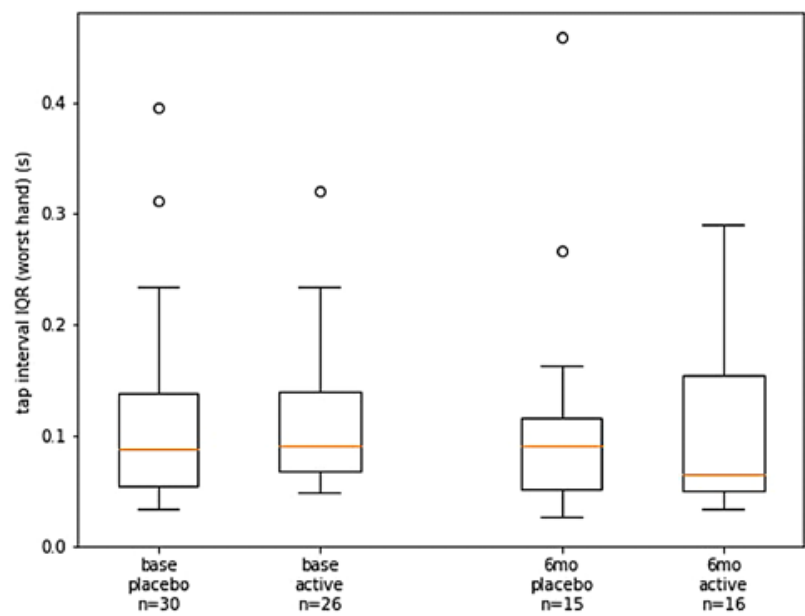

(b) Tapping variability in worst hand

Fig. 3. Tapping speed and variability in active (inosine) and placebo arms. a Tapping speed in best hand. b Tapping speed in worst hand. c Tapping variability in best hand. $\mathbf{d}$ Tapping variability in worst hand.

the total (parts I-III) MDS-UPDRS $\left(r_{\mathrm{s}}=-0.14\right)$. Tapping speed also correlated weakly with question 3.4 of the MDS-UPDRS on finger tapping $\left(r_{\mathrm{s}}=-0.19\right.$ for right hand and $r_{\mathrm{s}}=-0.22$ for the left hand). Variance in the tapping rate was moderately correlated with MDS-UPDRS part III, $r_{\mathrm{s}}=0.35$. Gait speed as measured by the smartphone application was mildly to moderately correlated with the motor $\left(r_{\mathrm{s}}=-0.25\right)$ and total $\left(r_{\mathrm{s}}=-0.34\right)$ MDS-UPDRS. The memory test correlated weakly with the MDS-UPDRS part I (nonmotor) score $\left(r_{\mathrm{s}}=-0.20\right)$.

\section{Impact of the Study Drug}

Over 6 months, there was no significant difference in finger tapping speed or variance between the drug and 


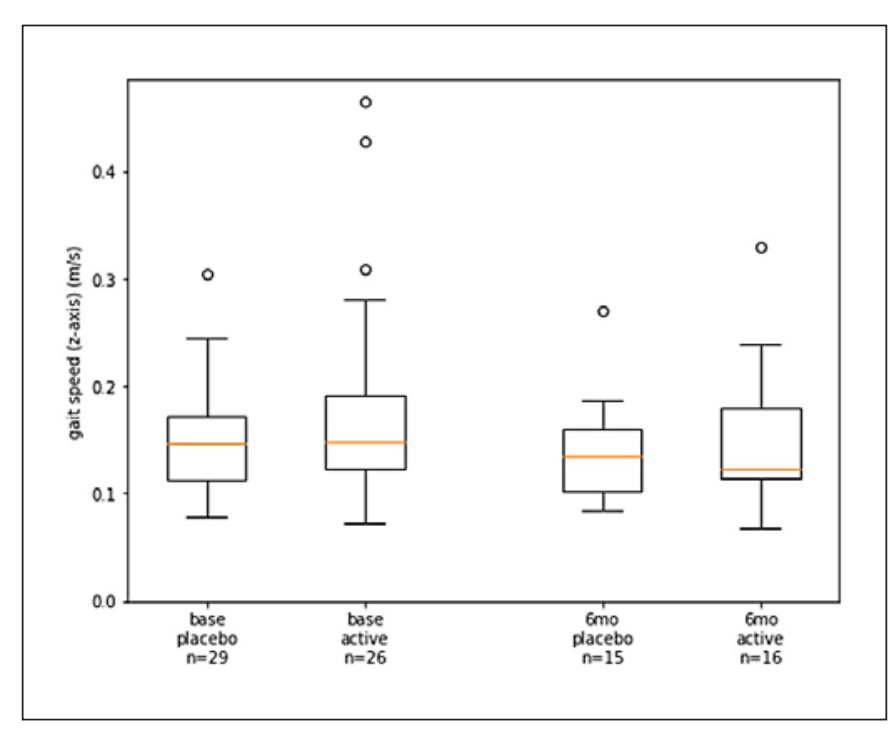

Fig. 4. Gait speed at baseline and month 6 among active smartphone participants.

placebo arms (Fig. 3). There was also no significant difference in gait speed between cohorts over this period (Fig. 4). No differences were detected in memory scores between the drug and placebo arms at either time point.

\section{Discussion}

In this pilot study, adherence to a smartphone research application in a phase 3 clinical trial declined as the study progressed, showed only modest correlation to traditional measures, and suggested that the active drug did not improve outcomes on the measures captured. While the promise of using digital tools to generate objective, sensitive, frequent assessments of disease and response to treatment is much discussed, this study highlights the need to be thoughtful and rigorous in their deployment. The relatively low adoption of the smartphone application and declining use as the trial progressed indicate that like other measures (e.g., biological and imaging), addition of digital tools needs to be planned prior to initiation of the study, outcome measures of interest predefined, and participants supported throughout the study. Previous studies have demonstrated widespread interest in use of these digital tools by the Parkinson's and other communities, but observational studies, like those of smartphone applications in general, have seen exponential declines in their use [20]. A recent phase I study that used a similar smartphone application found acceptable adher- ence with participants completing active tasks 3.5 times per week [3]. Other small research studies have demonstrated similar compliance [7]. Future efforts with digital measures may also seek to incorporate passive measures (e.g., step counts) of health that are less burdensome to participants than active ones. Additional measures, including of tremor, could also be of value in clinical trials $[21,22]$. In our case, introduction of the app midway through the trial to a limited set of participants, with limited site-level support for the app, lack of resources to track and encourage compliance with the app tasks, variability in the performance of the assessments, and premature termination of the study were likely the driving factors behind the modest results.

The measures evaluated in this study showed less correlation with traditional rating scales in contrast to a recent study with similar measures [3]. Some of the data captured from a smartphone (e.g., finger tapping) would be expected to correlate well with subjective assessments of similar tasks. However, much of the data captured by smartphones are underrepresented or not well assessed by the MDS-UPDRS. For example, gait impairment is a cardinal feature of the disease, but other than freezing, the motor examination only has one question (3.10) devoted to walking. Moreover, 2 of the 5 choices (slight and mild) will cover almost all individuals with early or even midstage Parkinson's disease. Activity and gait speed are important indicators of Parkinson's disease [23, 24] and overall health $[25,26]$. As such, smartphones and other digital measures can help supplement current measures in areas that might be excellent endpoints for clinical trials $[27,28]$. For example, regulatory authorities have recently accepted gait speed (for Duchenne muscular dystrophy) [29] and moderate-to-vigorous physical activity (for idiopathic pulmonary fibrosis) [30] as endpoints in clinical trials. The goal of new measures is not to replicate what is already present, but to measure new outcomes or current ones in advantageous ways (e.g., objectively, frequently, or in the real world). Therefore, strong correlations across parameters of various assessments may not be expected despite the parameters measuring similar domains of disease [31].

Consistent with the overall results of the phase 3 clinical trial [32], the smartphone measures in this pilot study found no evidence for the efficacy of inosine. These findings, however, are based on a small number of participants who were adherent to their use of the application over 6 months and did not look at changes over time. Additional factors, such as variability in adherence to and use of the application, could also have led to no change in 
the results as assessed by the smartphone. The 24-month study was also looking for longer term changes that might be seen with a disease-modifying therapy. That said, this pilot study is one of the first to use digital tools to assess the efficacy of a therapy in Parkinson's disease.

Despite the limitations of this study, the effort provides valuable guidance and lessons for future investigations that use smartphones or digital measures in future therapeutic trials: participation will be low and compliance will quickly diminish without vigorous participant engagement and support. As for other outcome measures, planning for digital measures should take place at the study's outset. If individuals are to use their own devices, data on those devices, software, and updates should be captured. Participants should be trained on the use and timing of assessments, and assessments (e.g., time of day and location of device) should be standardized to the extent possible. Regular monitoring of use and an easy way for the study team to contact participants (e.g., text and phone), and vice versa, are important to include. Where feasible, participants should be provided feedback on their results and use of the smartphone application. Investigators, sponsors, and regulators should recognize that digital measures may be capturing features of the disease that are not well ascertained by current measures, and thus correlations with traditional measures may be modest. Some of these digital endpoints may be novel (e.g., number of steps per day) but have enormous face validity and meaningfulness for multiple stakeholders, especially participants.

The need for real-world, objective measures of neurological and medical conditions is only expanding [11]. The COVID-19 pandemic has highlighted the need for these measures that can be captured outside of the clinic [33]. While this study demonstrated the growing pains of using new tools to measure Parkinson's disease, future studies will be better positioned to meet with greater success.

\section{Statement of Ethics}

Subjects provided their written informed consent to participate. The study protocol was reviewed and approved by institutional ethics review boards (University of Rochester Research Subjects Review Board, Reference \#58069).

\section{Conflict of Interest Statement}

Dr. L. Omberg has received research support from the National Institutes of Health/National Institute of Neurological Disorders and Stroke. Dr. M.A. Schwarzschild has been (or ex- pects to be) paid by the following commercial entities for service on a data monitoring committee (by Eli Lilly and Co.) and for service on scientific advisory boards both directly (by Prevail Therapeutics and Denali Therapeutics) and indirectly (via the Parkinson Study Group; by nQ Medical, Chase Therapeutics, Partner Therapeutics and Bial Biotech). He has also received royalty payments (via the Massachusetts General Hospital) for licensing of an adenosine A2A knockout mouse line. Dr. E.R. Dorsey has received honoraria for speaking at the American Neurological Association, Excellus BlueCross BlueShield, International Parkinson's and Movement Disorders Society, National Multiple Sclerosis Society, Northwestern University, Stanford University, Texas Neurological Society, and Weill Cornell; received compensation for consulting services from Abbott, Abbvie, Acadia, Acorda, Alzheimer's Drug Discovery Foundation, Ascension Health Alliance, Biogen, BluePrint Orphan, Clintrex, Curasen Therapeutics, DeciBio, Denali Therapeutics, Eli Lilly, Grand Rounds, Huntington Study Group, medical-legal services, Medical Communications Media, Medopad, Medrhythms, Michael J. Fox Foundation, MJH Holding LLC, NACCME, Olson Research Group, Origent Data Sciences, Otsuka, Pear Therapeutic, Praxis, Prilenia, Roche, Sanofi, Spark, Springer Healthcare, Sunovion Pharma, Sutter Bay Hospitals, Theravance, University of California Irvine, and WebMD; research support from Acadia Pharmaceuticals, Biogen, Biosensics, Burroughs Wellcome Fund, CuraSen, Greater Rochester Health Foundation, Huntington Study Group, Michael J. Fox Foundation, National Institutes of Health, Patient-Centered Outcomes Research Institute, Pfizer, PhotoPharmics, Safra Foundation, and Wave Life Sciences; editorial services for Karger Publications; and ownership interests with Grand Rounds (second opinion service). The remaining authors have no conflicts to report.

\section{Funding Sources}

The study was supported by a Burroughs Wellcome Fund Innovation in Regulatory Science Award, NIH Grant U01 NS107009, and the Farmer Family Foundation Parkinson's Research Initiative. These sponsors had no role in the preparation of the data or the manuscript.

\section{Author Contributions}

All authors provided critical revision of the manuscript for important intellectual content, provided final approval of the version to be published, and agreed to be accountable for all aspects of the work in ensuring that questions related to the accuracy or integrity of any part of the work are appropriately investigated and resolved. In addition to the foregoing, each individual author contributed as listed in the following: Alex Page contributed to analysis and interpretation of data and drafting of the manuscript. Norman Yung contributed to analysis and interpretation of data. Peggy Auinger contributed to analysis and interpretation of data. Charles Venuto contributed to analysis and interpretation of data. Alistair Glidden contributed to acquisition and interpretation of data. Eric Macklin contributed to analysis and interpretation of data. Larsson Omberg contributed to conception and design of the 
work and acquisition, analysis, and interpretation of data. Michael A. Schwarzschild contributed to conception and design of the work and acquisition and interpretation of data. E. Ray Dorsey contributed to conception and design of the work and interpretation of data and drafting of the manuscript.

\section{Data Availability Statement}

All data generated or analyzed during this study are included in this article. Further enquiries can be directed to the corresponding author.

\section{References}

1 Dorsey ER, Papapetropoulos S, Xiong M, Kieburtz K. The first frontier: digital biomarkers for neurodegenerative disorders. Digit Biomark. 2017;1:6-13.

2 Stamate C, Magoulas GD, Kueppers S, Nomikou E, Daskalopoulos I, Jha A, et al. The cloudUPDRS app: a medical device for the clinical assessment of Parkinson's disease. Pervasive Mob Comput. 2018;43:146-66.

3 Lipsmeier F, Taylor KI, Kilchenmann T, Wolf D, Scotland A, Schjodt-Eriksen J, et al. Evaluation of smartphone-based testing to generate exploratory outcome measures in a phase 1 Parkinson's disease clinical trial. Mov Disord. 2018;33:1287-97.

4 US-FDA. Submitting documents using realworld data and real-world evidence to FDA for drugs and biologics guidance for industry. 2019. Available from: https://www.fda.gov/ regulatory-information/search-fda-guidance-documents/submitting-documents-using-real-world-data-and-real-world-evidence-fda-drugs-and-biologics-guidance.

5 US-FDA. Use of real-world evidence to support regulatory decision-making for medical devices. 2017. Available from: https://www. fda.gov/regulatory-information/search-fdaguidance-documents/use-real-world-evidence-support-regulatory-decision-makingmedical-devices.

6 Printy BP, Renken LM, Herrmann JP, Lee I, Johnson B, Knight E, et al. 2014 36th Annual International Conference of the IEEE Engineering in Medicine and Biology Society. 2014. p. 2686-9.

7 Arora S, Venkataraman V, Zhan A, Donohue S, Biglan KM, Dorsey ER, et al. Detecting and monitoring the symptoms of Parkinson's disease using smartphones: a pilot study. Parkinsonism Relat Disord. 2015;21:650-3.

8 Ellis RJ, Ng YS, Zhu S, Tan DM, Anderson B, Schlaug G, et al. A validated smartphonebased assessment of gait and gait variability in Parkinson's disease. PLoS One. 2015;10: e0141694.

9 Espay AJ, Hausdorff JM, Sánchez-Ferro Á, Klucken J, Merola A, Bonato P, et al. A roadmap for implementation of patient-centered digital outcome measures in Parkinson's disease obtained using mobile health technologies. A roadmap for implementation of patient-centered digital outcome measures in Parkinson's disease obtained using mobile health technologies. Mov Disord. 2019;34(5): 657-63.

10 Perry B, Herrington W, Goldsack JC, Grandinetti CA, Vasisht KP, Landray MJ, et al. Use of mobile devices to measure outcomes in clinical research, 2010-2016: a systematic literature review. Digit Biomark. 2018;2:1130.

11 DiMe. DiMe's library of digital endpoints [accessed 2021 Dec 16]. https://www.dimesociety.org/communication-education/libraryof-digital-endpoints/.

12 Roche H-L. A study to evaluate the efficacy of prasinezumab (RO7046015/PRX002) in participants with early Parkinson's disease (PASADENA) [accessed 2021 Dec 16]. https:// clinicaltrials.gov/ct2/show/study/ NCT03100149.

13 Bot BM, Suver C, Neto EC, Kellen M, Klein A, Bare $\mathrm{C}$, et al. The mPower study, Parkinson disease mobile data collected using Research Kit. Sci Data. 2016;3:160011.

14 Schwarzschild MA. Study of urate elevation in Parkinson's disease. Phase 3 (SURE-PD3) [accessed 2021 Dec 16]. https://clinicaltrials. gov/ct2/show/NCT02642393.

15 NINDS. NINDS announces early study closure of SURE-PD3 trial [accessed 2021 Dec 16]. https://www.ninds.nih.gov/Disorders/ Clinical-Trials/Study-Urate-Elevation-Parkinsons-Disease-Phase-3-SURE-PD3.

16 Omberg L, Chaibub Neto E, Perumal TM, Pratap A, Tediarjo A, Adams J, et al. Remote smartphone monitoring of Parkinson's disease and individual response to therapy. Nat Biotechnol. 2021. Epub ahead of print.

17 Saez-Pons J, Stamate C, Weston D, Roussos G. Adjunct Proceedings of the 2019 ACM International Joint Conference on Pervasive and Ubiquitous Computing and Proceedings of the 2019 ACM International Symposium on Wearable Computers Association for Computing Machinery, London, UK. 2019. p. 939-43.

18 Tedjiaro A, Omberg L. Sage bionetworks gait feature extraction using PDKit \& rotation features [accessed 2021 Dec 16]. https:// github.com/arytontediarjo/PDKitRotationFeatures.

19 Pratap A, Neto EC, Snyder P, Stepnowsky C, Elhadad N, Grant D, et al. Indicators of retention in remote digital health studies: a crossstudy evaluation of 100,000 participants. NPJ Digit Med. 2020;3:21.

20 Dorsey ER, Yvonne Chan YF, McConnell MV, Shaw SY, Trister AD, Friend SH. The use of smartphones for health research. Acad Med. 2017;92:157-60.

21 Hssayeni MD, Jimenez-Shahed J, Burack MA, Ghoraani B. Wearable sensors for estimation of Parkinsonian tremor severity during free body movements. Sensors. 2019;19:4215.

22 Lonini L, Dai A, Shawen N, Simuni T, Poon C, Shimanovich L, et al. Wearable sensors for Parkinson's disease: which data are worth col- lecting for training symptom detection models. NPJ Digit Med. 2018;1:64

23 Cavanaugh JT, Ellis TD, Earhart GM, Ford MP, Foreman KB, Dibble LE. Capturing ambulatory activity decline in Parkinson's disease. J Neurol Phys Ther. 2012;36:51-7.

24 Paker N, Bugdayci D, Goksenoglu G Demircioğlu DT, Kesiktas N, Ince N. Gait speed and related factors in Parkinson's disease. J Phys Ther Sci. 2015;27:3675-9.

25 Saint-Maurice PF, Troiano RP, Bassett DR Jr, Graubard BI, Carlson SA, Shiroma EJ, et al. Association of daily step count and step intensity with mortality among US adults. JAMA. 2020;323:1151-60.

26 Studenski S, Perera S, Patel K, Rosano C, Faulkner K, Inzitari M, et al. Gait speed and survival in older adults. JAMA. 2011;305:50-

27 Wilson J, Alcock L, Yarnall AJ, Lord S, Lawson RA, Morris R, et al. Gait progression over 6 years in Parkinson's disease: effects of age, medication, and pathology. Front Aging Neurosci. 2020;12:577435.

28 Henderson EJ, Lord SR, Brodie MA, Gaunt DM, Lawrence AD, Close JC, et al. Rivastigmine for gait stability in patients with Parkinson's disease (ReSPonD): a randomised, double-blind, placebo-controlled, phase 2 trial. Lancet Neurol. 2016;15:249-58.

29 Haberkamp M, Moseley J, Athanasiou D, de Andres-Trelles F, Elferink A, Rosa MM, et al. European regulators' views on a wearable-derived performance measurement of ambulation for Duchenne muscular dystrophy regulatory trials. Neuromuscul Disord. 2019;29: 514-6.

30 Gisler S. FDA agrees to change study of Bellerophon's INOpulse for PH-ILD patients. 2019. Available from: https://pulmonaryhypertensionnews.com/2019/04/10/fda-agreesto-change-clinical-program-of-bellerophons-inopulse/.

31 Maetzler W, Rochester L, Bhidayasiri R, Espay AJ, Sánchez-Ferro A, van Uem JMT. Modernizing daily function assessment in Parkinson's disease using capacity, perception, and performance measures. Mov Disord. 2021;36(1):76-82.

32 Parkinson Study Group SURE-PD3 Investigators; Schwarzschild MA, Ascherio A, Casaceli C, Curhan GC, Fitzgerald R, et al. Effect of urate-elevating inosine on early parkinson disease progression: the SURE-PD3 randomized clinical trial. JAMA. 2021;326(10):926-39.

33 Goetz CG, Stebbins GT, Luo S. Movement Disorder Society-unified Parkinson's disease rating scale use in the COVID-19 era. Mov Disord. 2020;35:911 